\title{
Cause, Responsibility, and Blame: A Structural-Model Approach
}

\author{
Joseph Y. Halpern* \\ Cornell University \\ Dept. of Computer Science \\ Ithaca, NY 14853 \\ halpern@cs.cornell.edu \\ http://www.cs.cornell.edu/home/halpern
}

February 20, 2015

\begin{abstract}
A definition of causality introduced by Halpern and Pearl [2005a], which uses structural equations, is reviewed. A more refined definition is then considered, which takes into account issues of normality and typicality, which are well known to affect causal ascriptions. Causality is typically an all-or-nothing notion: either $A$ is a cause of $B$ or it is not. An extension of the definition of causality to capture notions of degree of responsibility and degree of blame, due to Chockler and Halpern [2004], is reviewed. For example, if someone wins an election 11-0, then each person who votes for him is less responsible for the victory than if he had won 6-5. Degree of blame takes into account an agent's epistemic state. Roughly speaking, the degree of blame of $A$ for $B$ is the expected degree of responsibility of $A$ for $B$, taken over the epistemic state of an agent. Finally, the structural-equations definition of causality is compared to Wright's [1985, 1988, 2001] NESS test.
\end{abstract}

${ }^{*}$ Supported in part by NSF under under grants ITR-0325453, IIS-0534064, and IIS-0911036, and by AFOSR under grant FA9550-05-1-0055. 


\section{Cause, Responsibility, and Blame: A Structural-Model Approach}

\section{Introduction}

It is generally agreed that the notion of legal cause is sorely in need of clarification. Not surprisingly, there has been a great deal of effort in the legal community to provide that clarification (see [Hart and Honoré 1985; Wright 1985; Wright 1988] and the references therein). Philosophers have also spent a great deal of effort attempting to clarify causality (see [Collins, Hall, and Paul 2004] for a recent collection of articles on the subject, along with pointers to the literature). Recently there has also been work on causality be computer scientists. It is that work that I report on here. In particular, I describe a definition of causality due to Halpern and Pearl [2005a]; I henceforth call this the HP definition.

The HP definition is more formal and mathematical than other definitions of causality in the literature. While this does add some initial overhead, it has the advantage of there being far less ambiguity. There is no need, as in most other definitions, to try to understand how to interpret the English (what counts as a "sufficient condition"? what is a "part" of a sufficient condition?). The first step in the HP definition involves building a formal model in which causality can be determined unambiguously. The definition will then say only that $A$ is a cause of $B$ in model $M$. It is possible to construct two closely related models $M_{1}$ and $M_{2}$ such that $A$ is a cause of $B$ in $M_{1}$ but not in $M_{2}$. There is not necessarily a "right" model (and, in any case, the definition is silent on what makes one model better than another, although see [Halpern and Hitchcock 2010] for some discussion of this issue). That means that, even if there is agreement regarding the definition of causality, there may be disagreement about which model better describes the real world. I view this as a feature of the definition. It moves the question of actual causality to the right arena-debating which of two (or more) models of the world is a better representation of those aspects of the world that one wishes to capture and reason about. This, indeed, is the type of debate that goes on in informal (and legal) arguments all the time.

The HP definition has several other advantages. For one thing, it can be used to provide a definition of explanation [Halpern and Pearl 2005b], an issue I do not discuss in this paper. For another, it can accommodate reasoning about normality and typicality, which is well known to affect causal ascriptions [Kahneman and Miller 1986]. A third advantage is that, as Chockler and Halpern [2004] showed, it can be extended to capture notions of degree of responsibility and degree of blame. To understand how this is done, first note that causality is an all-ornothing concept. That is, $A$ is either a cause of $B$ or it is not. As a consequence, thinking only in terms of causality prevents us from making what may be important distinctions. For example, suppose that Mr. B wins an election against Mr. G by a vote of 11-0. According to the HP definition, each of the people who voted for Mr. B is a cause of him winning. However, it seems that their degree of responsibility should not be as great as in the case when Mr. B wins 6-5. Chockler and Halpern suggested that the degree of responsibility of $A$ for $B$ should 
be taken to be $1 /(N+1)$, where $N$ is the minimal number of changes that have to be made to obtain a situation where $B$ counterfactually depends directly on $A$, one where, if $A$ hadn't happened, then $B$ wouldn't have happened. In the case of the 11-0 vote, each voter has degree of responsibility 1/6 for Mr. B's victory, since the votes of five other voters have to be changed (giving a vote of 6-5) before Mr. B's victory counterfactually depends directly on the vote of any of the six remaining people who voted for him; if any of these six voters had changed their vote, then Mr. G would have won. By way of contrast, in the case of a 6-5 vote, each of the six voters is already directly counterfactually responsible for Mr. B's victory; if any of them had changed their vote, then Mr. B would not have won. Thus, each has degree of responsibility 1. In general, the degree of responsibility of $A$ for $B$ is between 0 and 1 . It is 0 if and only if $A$ is not a cause of $B$; it is 1 if $B$ directly counterfactually depends on $A$; it is strictly between 0 and 1 otherwise.

This definition of degree of responsibility implicitly assumes that the facts of the world and how the world works are known. For example, when saying that voter 1 has degree of responsibility $1 / 6$ for Mr. B's win when the vote is $11-0$, we assume that the vote and the procedure for determining a winner (majority wins) is known. There is no uncertainty about this. But this misses out on important component of determining what Chockler and Halpern called blame: the epistemic state. Consider a doctor who treats a patient with a particular drug resulting in the patient's death. The doctor's treatment is a cause of the patient's death; indeed, the doctor may well bear degree of responsibility 1 for the death. However, if the doctor had no idea that the treatment had adverse side effects for people with high blood pressure, he should perhaps not be held to blame for the death. Actually, in legal arguments, it may not be so relevant what the doctor actually did or did not know, but what he should have known. Thus, rather than considering the doctor's actual epistemic state, it may be more important to consider what his epistemic state should have been. Chocker and Halpern [2004] give a definition of degree of blame that takes this into account; roughly speaking, the degree of blame of an agent who performed $A$ has for $B$ is the expected degree of responsibility of $A$ for $B$, relative to agent's epistemic state.

To understand the difference between responsibility and blame, suppose that there is a firing squad consisting of ten excellent marksmen. Only one of them has live bullets in his rifle; the rest have blanks. The marksmen do not know which of them has the live bullets. The marksmen shoot at the prisoner and he dies. The only marksman that is the cause of the prisoner's death is the one with the live bullets. That marksman has degree of responsibility 1 for the death; all the rest have degree of responsibility 0 . However, each of the marksmen has degree of blame $1 / 10$.

The rest of this paper is organized as follows. In Section 2, I provide a gentle introduction to structural equations and causal models. In Section 3, I review the HP definition of actual causality. In Section 4, I show how the definition can be refined to deal with normality and typicality. In Section 5, I show how the definition can be extended to capture responsibility and blame. In Section 6, I compare the structural-model definition of causality is compared to Wright's [1985, 1988, 2001] NESS test, which is perhaps the closest work in the legal literature to it. I conclude in Section 7. Sections 2 and 3 are largely taken from [Halpern and 
Pearl 2005a]; Section 4 is based on material from [Halpern and Hitchcock 2013]; Section 5 is based on material from [Chockler and Halpern 2004]; and material in Section 6 appeared in preliminary form in [Halpern 2008]. The reader is encouraged to consult these papers for more details, more intuition, and more examples.

\section{Causal Models}

In this section, I review the formal model of causality used in the HP definition. The description of causal models given here is taken from [Halpern 2000]; it is a formalization of earlier work of Pearl [1995], further developed in [Galles and Pearl 1997; Halpern 2000; Pearl 2000].

The model assumes that the world is described in terms of random variables and their values. For example, if we are trying to determine whether a forest fire was caused by lightning or an arsonist, we can take the world to be described by three random variables:

- $F F$ for forest fire, where $F F=1$ if there is a forest fire and $F F=0$ otherwise;

- $L$ for lightning, where $L=1$ if lightning occurred and $L=0$ otherwise;

- $M D$ for match dropped (by arsonist), where $M D=1$ if the arsonist dropped a lit match, and $M D=0$ otherwise.

Similarly, in the case of the 11-0 vote, we can describe the world by 12 random variables, $V_{1}, \ldots, V_{11}, W$, where $V_{i}=0$ if voter $i$ voted for Mr. B and $V_{1}=1$ if voter $i$ voted for Mr. G, for $i=1, \ldots, 11, W=0$ if Mr. B wins, and $W=1$ if Mr. G wins. In these two examples, all the random variables are binary, that is, they take on only two values. There is no problem allowing a random variable to have more than two possible values. For example, the random variable $V_{i}$ could be either 0,1 , or 2 , where $V_{i}=2$ if $i$ does not vote; similarly, we could take $W=2$ if the vote is tied, so neither candidate wins.

The choice of random variables determines the language used to frame the situation. Although there is no "right" choice, clearly some choices are more appropriate than others. For example, when trying to determine the cause of Sam's lung cancer, if there is no random variable corresponding to smoking in a model then, in that model, we will not be able to conclude that smoking is a cause of Sam's lung cancer.

Some random variables may have a causal influence on others. This influence is modeled by a set of structural equations. For example, if we want to model the fact that if the arsonist drops a match or lightning strikes then a fire starts, we could use the random variables $M D$, $F F$, and $L$ as above, with the equation $F F=\max (L, M D)$; that is, the value of the random variable $F F$ is the maximum of the values of the random variables $M D$ and $L$. This equation says, among other things, that if $M D=0$ and $L=1$, then $F F=1$. The equality sign in this equation should be thought of more like an assignment statement in programming languages; once we set the values of $M D$ and $L$, then the value of $F F$ is set to their maximum. However, 
despite the equality, if a forest fire starts some other way, that does not force the value of either $M D$ or $L$ to be 1 .

Alternatively, if we want to model the fact that a fire requires both a lightning strike and a dropped match (perhaps the wood is so wet that it needs two sources of fire to get going), then the only change in the model is that the equation for $F F$ becomes $F F=\min (L, M D)$; the value of $F F$ is the minimum of the values of $M D$ and $L$. The only way that $F F=1$ is if both $L=1$ and $M D=1$.

Both of these models are somewhat simplistic. Lightning does not always result in a fire, nor does dropping a lit match. One way of dealing with this would be to make the assignment statements probabilistic. For example, we could say that the probability that $F F=1$ conditional on $L=1$ is .8. This approach would lead to rather complicated definitions. It is much simpler to think of all the equations as being deterministic and, intuitively, use enough variables to capture all the conditions that determine whether there is a forest fire. One way to do this is simply to add those variables explicitly. For example, we could add variables that talk about the dryness of the wood, the amount of undergrowth, the presence of sufficient oxygen (fires do not start so easily on the top of high mountains), and so on. If a modeler does not want to add all these variables explicitly (the details may simply not be relevant to the analysis), another alternative is to use a single variable, say $U$, which intuitively incorporates all the relevant factors, without describing them explicitly. The value of $U$ would determine whether the lightning strikes, whether the match is dropped by the arsonist, and whether both are needed to start a fire, just one, or neither (perhaps fires start spontaneously, or there is a cause not modeled by the random variables used). In this way of modeling things, $U$ would take on eight possible values of the form $(i, j, k)$, where $i, j$, and $k$ are each either 0 or 1 . Intuitively, $i$ describes whether the external conditions are such that the lightning strikes (and encapsulates all the conditions, such as humidity and temperature, that affect whether the lightning strikes); $j$ describes whether the arsonist drops the match (and thus encapsulates the psychological conditions that determine whether the arsonist drops the match); and $k$ describes whether just one of or both a dropped match and lightning are needed for the forest fire. Thus, the equation could say that $F F=1$ if, for example, $U=(1,1,1)$ (so that the lightning strikes, the match is dropped, and only one of them is needed to have a fire) or if $U=(1,1,2)$, but not if $U=(1,0,2)$. For future reference, let $U_{1}, U_{2}$, and $U_{3}$ denote the three components of the value of $U$ in this example, so that if $U=(i, j, k)$, then $U_{1}=i, U_{2}=j$, and $U_{3}=k$.

It is conceptually useful to split the random variables into two sets, the exogenous variables, whose values are determined by factors outside the model, and the endogenous variables, whose values are ultimately determined by the exogenous variables. In the forest-fire example, the variable $U$ is exogenous, while the variables $F F, L$, and $M D$ are endogenous. We want to take as given that there is enough oxygen for the fire, that the wood is sufficiently dry to burn, and whatever factors make the arsonist drop the match. Thus, it is only the endogenous variables whose values are described by the structural equations.

With this background, we can formally define a causal model $M$ as a pair $(\mathcal{S}, \mathcal{F})$, where $\mathcal{S}$ is a signature, which explicitly lists the endogenous and exogenous variables and characterizes 
their possible values, and $\mathcal{F}$ defines a set of modifiable structural equations, relating the values of the variables. In the next two paragraphs, I define $\mathcal{S}$ and $\mathcal{F}$ formally; the definitions can be skipped by the less mathematically inclined reader.

A signature $\mathcal{S}$ is a tuple $(\mathcal{U}, \mathcal{V}, \mathcal{R})$, where $\mathcal{U}$ is a set of exogenous variables, $\mathcal{V}$ is a set of endogenous variables, and $\mathcal{R}$ associates with every variable $Y \in \mathcal{U} \cup \mathcal{V}$ a nonempty set $\mathcal{R}(Y)$ of possible values for $Y$ (i.e., the set of values over which $Y$ ranges). As suggested above, in the forest-fire example, we have $\mathcal{U}=\{U\}, \mathcal{R}(U)$ consists of the 8 possible values of $U$ discussed earlier, $\mathcal{V}=\{F F, L, M D\}$, and $\mathcal{R}(F F)=\mathcal{R}(L)=\mathcal{R}(M D)=\{0,1\}$.

$\mathcal{F}$ associates with each endogenous variable $X \in \mathcal{V}$ a function denoted $F_{X}$ such that $F_{X}$ maps $\times_{Z \in(\mathcal{U} \cup \mathcal{V}-\{X\})} \mathcal{R}(Z)$ to $\mathcal{R}(X) .{ }^{1}$ This mathematical notation just makes precise the fact that $F_{X}$ determines the value of $X$, given the values of all the other variables in $\mathcal{U} \cup \mathcal{V}$. In the running forest-fire example, we would have $F_{F F}(L, M D, U)=1$ if $U_{3}=1$ and $\max (L, M D)=1$ (so that at least one of $L$ or $M D$ is 1 ); similarly, $F_{F F}(L, M D, U)=1$ if $U_{3}=2$ and $\min (L, M D)=1$ (so that both $L$ and $M D$ are 1). Note that the value of $F_{F F}$ is independent of the first two components of $U$; all that matters is $U_{3}$ and the values of $L$ and $M D$. The first two components of $U$ are used in the equations for $L$ and $M D: U_{1}$ determines the value of $L$ and $U_{2}$ determines the value of $M D$; specifically, $F_{L}(M D, F F, U)=U_{1}$ and $F_{M D}(L, F F, U)=U_{2}$. Note that the values of $L$ and $M D$ are independent of the value of $F F$.

I sometimes simplify notation and write $X=Y+U$ instead of $F_{X}\left(Y, Y^{\prime}, U\right)=Y+U$. With this simplified notation, the equations for the forest-fire example become

$$
\begin{aligned}
L & =U_{1} \\
M D & =U_{2} \\
F F & = \begin{cases}\max (L, M D) & \text { if } U_{3}=1 \\
\min (L, M D) & \text { if } U_{3}=2 .\end{cases}
\end{aligned}
$$

Although I may write something like $X=Y+U$, I stress that the fact that $X$ is assigned $Y+U$ does not imply that $Y$ is assigned $X-U$; that is, $F_{Y}(X, Z, U)=X-U$ does not necessarily hold. With this equation, if $Y=3$ and $U=2$, then $X=5$, regardless of how $Y^{\prime}$ is set. It is this asymmetry in the treatment of the variables on the left- and right-hand side of the equality sign that arguably leads to the asymmetry of causality: if $A$ is a cause of $B$, it will not be the case that $B$ is a cause of $A$.

The key role of the structural equations is to define what happens in the presence of external interventions. For example, they describe what happens if the arsonist does not drop the match. In this case, there is a forest fire exactly if there is lightning and lightning by itself suffices for a fire; that is, if the exogenous variable $U$ has the form $(i, 1,1)$. Understanding the effect of interventions will be critical in defining causality.

Setting the value of some variable $X$ to $x$ in a causal model $M=(\mathcal{S}, \mathcal{F})$ results in a new causal model denoted $M_{X \leftarrow x}$. In the new causal model, the equation for $X$ is very simple: $X$ is just set to $x$; the remaining equations are unchanged. More formally, $M_{X \leftarrow x}=\left(\mathcal{S}, \mathcal{F}_{X \leftarrow x}\right)$,

\footnotetext{
${ }^{1}$ Recall that $\mathcal{U} \cup \mathcal{V}-\{X\}$ is the set consisting of all the variables in either $\mathcal{U}$ or $\mathcal{V}$ that are not in $\{X\}$.
} 
where $\mathcal{F}_{X \leftarrow x}$ is the result of replacing the equation for $X$ in $\mathcal{F}$ by $X=x$. Thus, if $M$ is the causal model for the forest-fire example, then in $M_{M D \leftarrow 0}$, the model where the arsonist does not drop the match, the equation $M D=U_{2}$ is replaced by $M D=0$.

Counterfactuals (statements counter to fact) have often been considered when trying to define causality. These (causal) equations can be given a straightforward counterfactual interpretation. An equation such as $x=F_{X}(u, y)$ should be thought of as saying that in a context where the exogenous variable $U$ has value $u$, if $Y$ were set to $y$ by some means (not specified in the model), then $X$ would take on the value $x$, as dictated by $F_{X}$. However, if the value of $X$ is set by some other means (e.g., the forest catches fire due to a volcano eruption), then the assignment specified by $F_{X}$ is "overruled"; $Y$ is no longer committed to tracking $X$ according to $F_{X}$. This emphasizes the point that variables on the left-hand side of equations are treated differently from ones on the right-hand side.

In the philosophy community, counterfactuals are typically defined in terms of "closest worlds" [Lewis 1973; Stalnaker 1968]; a statement of the form "if $A$ were the case then $B$ would be true" is taken to be true if in the "closest world(s)" to the actual world where $A$ is true, $B$ is also true. This modification of equations may be given a simple "closest world" interpretation: the solution of the equations obtained by replacing the equation for $Y$ with the equation $Y=y$, while leaving all other equations unaltered, gives the closest world to the actual world where $Y=y$. (See [Halpern 2009] for further discussion of the relationship between causal models and closest worlds.) The asymmetry embodied in the structural equations can be understood in terms of closest worlds. If either the match or the lightning suffice to start the fire, then in the closest world to the actual world where a lit match is dropped the forest burns down. However, it is not necessarily the case that in the world closest to one where the forest burns down a lit match is dropped.

It may seem somewhat circular to use causal models, which clearly already encode causal relationships, to define causality. Nevertheless, as we shall see, there is no circularity. In most examples, there is general agreement as to the appropriate causal model. The structural equations do not express actual causality; rather, they express the effects of interventions. Of course, there may be uncertainty about the effects of interventions, just as there may be uncertainty about the true setting of the values of the exogenous variables in a causal model. For example, we may be uncertain about whether smoking causes cancer (this represents uncertainty about the causal model) and uncertain about whether a particular patient actually smoked (this is uncertainty about the value of the exogenous variable that determines whether the patient smokes). This uncertainty can be described by putting a probability on causal models and on the values of the exogenous variables. We can then talk about the probability that $A$ is a cause of $B$. (See Section 5.2 for further discussion of this point.)

In a causal model, it is possible that the value of $X$ depends on the value of $Y$ (i.e., the equation $F_{X}$ is such that changes in $Y$ can change the value of $X$ ) and the value of $Y$ depends on the value of $X$. Intuitively, this says that $X$ can potentially affect $Y$ and that $Y$ can potentially affect $X$. While allowed by the framework, this type of situation does not typically happen in examples of interest. Even when it seems to happen, we can often recast the problem 
using a different (and arguably more appropriate)) choice of variables so that we do not have such cyclic dependence (see [Halpern and Pearl 2005a] for an example). Since dealing with such cyclic dependence would complicate the exposition, I restrict attention here to what are called recursive (or acyclic) models. This is the special case where there is some total ordering $\prec$ of the endogenous variables (the ones in $\mathcal{V}$ ) such that if $X \prec Y$, then $X$ is independent of $Y$, that is, $F_{X}(\ldots, y, \ldots)=F_{X}\left(\ldots, y^{\prime}, \ldots\right)$ for all $y, y^{\prime} \in \mathcal{R}(Y)$. Intuitively, if a theory is recursive, there is no feedback. If $X \prec Y$, then the value of $X$ may affect the value of $Y$, but the value of $Y$ cannot affect the value of $X$.

It should be clear that if $M$ is an acyclic causal model, then given a context, that is, a setting $\vec{u}$ for the exogenous variables in $\mathcal{U}$, there is a unique solution for all the equations. ${ }^{2}$ We simply solve for the variables in the order given by $\prec$. The value of the variables that come first in the order, that is, the variables $X$ such that there is no variable $Y$ such that $Y \prec X$, depend only on the exogenous variables, so their value is immediately determined by the values of the exogenous variables. The values of values later in the order can be determined once we have determined the values of all the variables earlier in the order.

There are many nontrivial decisions to be made when choosing the structural model to describe a given situation. One significant decision is the set of variables used. As we shall see, the events that can be causes and those that can be caused are expressed in terms of these variables, as are all the intermediate events. The choice of variables essentially determines the "language" of the discussion; new events cannot be created on the fly, so to speak. In our running example, the fact that there is no variable for unattended campfires means that the model does not allow us to consider unattended campfires as a cause of the forest fire.

Once the set of variables is chosen, the next step is to decide which are exogenous and which are endogenous. As I said earlier, the exogenous variables to some extent encode the background situation that we want to take for granted. Other implicit background assumptions are encoded in the structural equations themselves. Suppose that we are trying to decide whether a lightning bolt or a match was the cause of the forest fire, and we want to take for granted that there is sufficient oxygen in the air and the wood is dry. We could model the dryness of the wood by an exogenous variable $D$ with values 0 (the wood is wet) and 1 (the wood is dry). ${ }^{3}$ By making $D$ exogenous, its value is assumed to be given and out of the control of the modeler. We could also take the amount of oxygen to be described by an exogenous variable (for example, there could be a variable $O$ with two values - 0 , for insufficient oxygen, and 1, for sufficient oxygen); alternatively, we could choose not to model oxygen explicitly at all. For example, suppose that we have, as before, a random variable $M D$ for match dropped by arsonist, and another variable $W B$ for wood burning, with values 0 (it's not) and 1 (it is). The structural equation $F_{W B}$ would describe the dependence of $W B$ on $D$ and $M D$. By setting

\footnotetext{
${ }^{2} \mathrm{~A}$ note on notation: in general, there may be several exogenous variables in a causal model, not just one. The "vector" notation $\vec{u}$ is used here and elsewhere to denote the values of all of them. For example, if there are three exogenous variables, say $U_{1}, U_{2}$, and $U_{3}$, and $U_{1}=0, U_{2}=0$, and $U_{3}=1$, then $\vec{u}$ is $(0,0,1)$. This vector notation is also used to describe the values $\vec{x}$ of a collection $\vec{X}$ of endogenous variables.

${ }^{3}$ Of course, in practice, we may want to allow $D$ to have more values, indicating the degree of dryness of the wood, but that level of complexity is unnecessary for the points that I am trying to make here.
} 
$F_{W B}(1,1)=1$, we are saying that the wood will burn if the lit match is dropped and the wood is dry. Thus, the equation is implicitly modeling our assumption that there is sufficient oxygen for the wood to burn.

According to the definition of actual cause in Section 3, only endogenous variables can be causes or be caused. Thus, if no variables encode the presence of oxygen, or if it is encoded only in an exogenous variable, then oxygen cannot be a cause of the forest burning. If we were to explicitly model the amount of oxygen in the air (which certainly might be relevant if we were analyzing fires on Mount Everest), then $F_{W B}$ would also take values of $O$ as an argument, and the presence of sufficient oxygen might well be a cause of the wood burning, and hence the forest burning. ${ }^{4}$ Interestingly, in the law, there is a distinction between what are called conditions and causes [Katz 1987]. Under typical circumstances, the presence of oxygen would be considered a condition, and would thus not count as a cause of the forest burning, while the lightning would. While the distinction is considered important, it does not seem to have been carefully formalized. One way of understanding it is in terms of exogenous vs. endogenous variables; conditions are exogenous, (potential) causes are endogenous. I discuss a complementary approach to understanding it, in terms of theories of normality, in Section 4.

It is not always straightforward to decide what the "right" choice of variables is, or, more generally, what the "right" causal model is in a given situation; nor is it always obvious which of two causal models is "better" in some sense. These decisions often lie at the heart of determining actual causality in the real world. Disagreements about causal relationships often boil down to disagreements about the causal model. While the formalism presented here does not provide techniques to settle disputes about which causal model is the right one, at least it provides tools for carefully describing the differences between causal models, so that it should lead to more informed and principled decisions about those choices (see [?; Halpern and Hitchcock 2010] for more discussion of these points).

\section{A Formal Definition of Actual Cause}

\subsection{A language for describing causes}

To make the definition of actual causality precise, it is helpful to have a formal language for making statements about causality. The language is a slight extension of propositional logic, now often taught in highschool. Given a signature $\mathcal{S}=(\mathcal{U}, \mathcal{V}, \mathcal{R})$, a primitive event is a formula of the form $X=x$, for $X \in \mathcal{V}$ and $x \in \mathcal{R}(X)$. That is, $X$ is an endogenous variable, and $x$ is a possible value of $X$. The primitive event $M D=0$ says "the lit match is not dropped"; the primitive event $L=1$ says "lightning occurred". As in propositional logic, the symbols $\wedge, \vee$, and $\neg$ are used to denote conjunction, disjunction, and negation, respectively. Thus, the formula $M D=0 \vee L=1$ says "either the lit match is not dropped or lightning occurred", $M D=0 \wedge L=1$ says "either the lit match is not dropped and lightning occurred", and $\neg(L=$

\footnotetext{
${ }^{4}$ Of course, $F_{W B}$ might take yet other variables as arguments.
} 
1) says "lightning did not occur" (which is equivalent to $L=0$, given that the only possible values of $L$ are 0 or 1). A Boolean combination of primitive events is a formula that is obtained by combining primitive events using $\wedge, \vee$, and $\neg$. Thus, $\neg(M D=0 \vee L=1) \wedge W B=1$ is a Boolean combination of the primitive events $M D=0, L=1$, and $W B=1$.

A causal formula (over $\mathcal{S}$ ) is one of the form $\left[Y_{1} \leftarrow y_{1}, \ldots, Y_{k} \leftarrow y_{k}\right] \varphi$, where

- $\varphi$ is a Boolean combination of primitive events,

- $Y_{1}, \ldots, Y_{k}$ are distinct variables in $\mathcal{V}$, and

- $y_{i} \in \mathcal{R}\left(Y_{i}\right)$.

Such a formula is abbreviated as $[\vec{Y} \leftarrow \vec{y}] \varphi$. The special case where $k=0$ is abbreviated as $\varphi$. Intuitively, $\left[Y_{1} \leftarrow y_{1}, \ldots, Y_{k} \leftarrow y_{k}\right] \varphi$ says that $\varphi$ would hold if $Y_{i}$ were set to $y_{i}$, for $i=1, \ldots, k$.

A causal formula $\psi$ is true or false in a causal model, given a context. I write $(M, \vec{u}) \models \psi$ if the causal formula $\psi$ is true in causal model $M$ given context $\vec{u}$. Perhaps not surprisingly, $(M, \vec{u}) \models \psi$ is read " $\psi$ is true in context $\vec{u}$ in causal model model $M$ ". $(M, \vec{u}) \models$ $[\vec{Y} \leftarrow \vec{y}](X=x)$ if the variable $X$ has value $x$ in the unique (since we are dealing with acyclic models) solution to the equations in $M_{\vec{Y} \leftarrow \vec{y}}$ in context $\vec{u}$ (i.e., the unique vector of values for the endogenous variables that simultaneously satisfies all equations in $\mathcal{F}_{\vec{Y} \leftarrow \vec{y}}$ with the variables in $\mathcal{U}$ set to $\vec{u})$. $(M, \vec{u}) \models[\vec{Y} \leftarrow \vec{y}] \varphi$ for an arbitrary Boolean combination $\varphi$ of formulas of the form $\vec{X}=\vec{x}$ is defined similarly. I write $M \models \varphi$ if $(M, \vec{u}) \models \varphi$ for all contexts $\vec{u}$.

For example, if $M$ is the causal model described earlier for the forest fire, then $(M,(1,1,1)) \models$ $[M D \leftarrow 0](F F=1)$, since even if the arsonist is somehow prevented from dropping the match, the forest burns (thanks to the lightning); similarly, $(M,(1,1,1)) \models[L \leftarrow 0](F F=1)$. However, $(M,(1,1,1)) \models[L \leftarrow 0 ; M D \leftarrow 0](F F=0)$ : if arsonist does not drop the lit match and the lightning does not strike, then forest does not burn. Moreover, $(M,(1,1,2)) \models$ $[M D \leftarrow 0](F F=1)$; if both lightning and the match are needed for the forest to burn down, then if the arsonist does not drop the match, the forest does not burn down.

\subsection{A preliminary definition of causality}

The HP definition of causality, like many others, is based on counterfactuals. The idea is that $A$ is a cause of $B$ if, if $A$ hadn't occurred (although it did), then $B$ would not have occurred. This idea goes back to at least Hume [1748, Section VIII], who said:

We may define a cause to be an object followed by another, ..., if the first object had not been, the second never had existed.

This is essentially the but-for test, perhaps the most widely used test of actual causation in tort adjudication. The but-for test states that an act is a cause of injury if and only if, but for the act (i.e., had the act not occurred), the injury would not have occurred. 
There are two well-known problems with this definition. The first can be seen by considering the forest-fire example again. Suppose that an arsonist drops a match and lightning strikes, and either one suffices to start the fire. Which is the cause? According to a naive interpretation of the counterfactual definition, neither is. If the match hadn't dropped, then the lightning would still have struck, so there would have been a forest fire anyway. Similarly, if the lightning had not occurred, there still would have been a forest fire. Our definition will declare both lightning and the arsonist cases of the fire. (In general, there may be more than one cause of an outcome.)

A more subtle problem is what philosophers have called preemption, where there are two potential causes of an event, one of which preempts the other. Preemption is illustrated by the following story, taken from [Hall 2004]:

Suzy and Billy both pick up rocks and throw them at a bottle. Suzy's rock gets there first, shattering the bottle. Since both throws are perfectly accurate, Billy's would have shattered the bottle had it not been preempted by Suzy's throw.

Common sense suggests that Suzy's throw is the cause of the shattering, but Billy's is not. However, Suzy's throw is not a cause according to the naive counterfactual definition; if Suzy hadn't thrown, then Billy's throw would have shattered the bottle.

The HP definition deals with the first problem by defining causality as counterfactual dependency under certain contingencies. In the forest-fire example, the forest fire does counterfactually depend on the lightning under the contingency that the arsonist does not drop the match; similarly, the forest fire depends counterfactually on the arsonist's match under the contingency that the lightning does not strike. Clearly we need to be a little careful here to limit the contingencies that can be considered. We do not want to make Billy's throw the cause of the bottle shattering by considering the contingency that Suzy does not throw. The reason that we consider Suzy's throw to be the cause and Billy's throw not to be the cause is that Suzy's rock hit the bottle, while Billy's did not. Somehow the definition must capture this obvious intuition.

With this background, HP's preliminary definition of causality can be given; it is quite close to the final definition, given in Section 4. The types of events that the HP definition allows as actual causes are ones of the form $X_{1}=x_{1} \wedge \ldots \wedge X_{k}=x_{k}$-that is, conjunctions of primitive events; this is often abbreviated as $\vec{X}=\vec{x}$. The events that can be caused are arbitrary Boolean combinations of primitive events. The definition does not allow statements of the form " $A$ or $A^{\prime}$ is a cause of $B$," although this could be treated as being equivalent to "either $A$ is a cause of $B$ or $A^{\prime}$ is a cause of $B$ ". On the other hand, statements such as " $A$ is a cause of $B$ or $B^{\prime \prime}$ " are allowed; this is not equivalent to "either $A$ is a cause of $B$ or $A$ is a cause of $B^{\prime \prime}$.

Definition 3.1: (Actual cause; preliminary version) $\vec{X}=\vec{x}$ is an actual cause of $\varphi$ in $(M, \vec{u})$ if the following three conditions hold:

AC1. $(M, \vec{u}) \models(\vec{X}=\vec{x})$ and $(M, \vec{u}) \models \varphi$. 
AC2. There is a partition of $\mathcal{V}$ (the set of endogenous variables) into two subsets $\vec{Z}$ and $\vec{W}$ with $\vec{X} \subseteq \vec{Z}$ and a setting $\vec{x}^{\prime}$ and $\vec{w}$ of the variables in $\vec{X}$ and $\vec{W}$, respectively, such that if $(M, \vec{u}) \models Z=z^{*}$ for all $Z \in \vec{Z}$, then both of the following conditions hold:

(a) $(M, \vec{u})=\left[\vec{X} \leftarrow \vec{x}^{\prime}, \vec{W} \leftarrow \vec{w}\right] \neg \varphi$.

(b) $(M, \vec{u})=\left[\vec{X} \leftarrow \vec{x}, \vec{W}^{\prime} \leftarrow \vec{w}, \vec{Z}^{\prime} \leftarrow \vec{z}^{*}\right] \varphi$ for all subsets $\vec{W}^{\prime}$ of $\vec{W}$ and all subsets $\vec{Z}^{\prime}$ of $\vec{Z}$, where I abuse notation and write $\vec{W}^{\prime} \leftarrow \vec{w}$ to denote the assignment where the variables in $\vec{W}^{\prime}$ get the same values as they would in the assignment $\vec{W} \leftarrow \vec{w}$ (thus, components in the vector $\vec{w}$ that do not match any variable in $\vec{W}^{\prime}$ are ignored).

AC3. $\vec{X}$ is minimal; no subset of $\vec{X}$ satisfies conditions AC1 and AC2.

AC1 is just says that $\vec{X}=\vec{x}$ cannot be considered a cause of $\varphi$ unless both $\vec{X}=\vec{x}$ and $\varphi$ actually happen. AC3 is a minimality condition, which ensures that only those elements of the conjunction $\vec{X}=\vec{x}$ that are essential for changing $\varphi$ in AC2(a) are considered part of a cause; inessential elements are pruned. Without AC3, if dropping a lit match qualified as a cause of the forest fire, then dropping a match and sneezing would also pass the tests of AC1 and AC2. AC3 serves here to strip "sneezing" and other irrelevant, over-specific details from the cause.

Clearly, all the "action" in the definition occurs in AC2. We can think of the variables in $\vec{Z}$ as making up the "causal path" from $\vec{X}$ to $\varphi$. Intuitively, changing the value of some variable in $\vec{X}$ results in changing the value(s) of some variable(s) in $\vec{Z}$, which results in the values of some other variable(s) in $\vec{Z}$ being changed, which finally results in the value of $\varphi$ changing. The remaining endogenous variables, the ones in $\vec{W}$, are off to the side, so to speak, but may still have an indirect effect on what happens. AC2(a) is essentially the standard counterfactual definition of causality, but with a twist. If we want to show that $\vec{X}=\vec{x}$ is a cause of $\varphi$, we must show (in part) that if $\vec{X}$ had a different value, then so too would $\varphi$. However, this effect of $\vec{X}$ on the value of $\varphi$ may not hold in the actual context; the value of $\vec{W}$ may have to be different to allow the effect to manifest itself. For example, in the context where both the lightning strikes and the arsonist drops a match, stopping the arsonist from dropping the match will not prevent the forest fire. The counterfactual effect of the arsonist on the forest fire manifests itself only in a situation where the lightning does not strike (i.e., where $L$ is set to 0 ).

AC2(b) is perhaps the most complicated condition. It limits the "permissiveness" of AC2(a) with regard to the contingencies (i.e., the values of the variables in $\vec{W}$ ) that can be considered. Essentially, it ensures that $\vec{X}$ alone suffices to bring about the change from $\varphi$ to $\neg \varphi$; setting $\vec{W}$ to $\vec{w}$ merely eliminates possibly spurious side effects that may mask the effect of changing the value of $\vec{X}$. Moreover, although the values of variables on the casual path (i.e., the variables $\vec{Z})$ may be perturbed by the change to $\vec{W}$, this perturbation has no impact on the value of $\varphi$. Note that if $(M, \vec{u}) \models \vec{Z}=\vec{z}^{*}$, then $\vec{z}^{*}$ is the value of the variables in $\vec{Z}$ in the context $\vec{u}$. We capture the fact that the perturbation has no impact on the value of $\varphi$ by saying that if some variables $\vec{Z}^{\prime}$ on the causal path were set to their original values in the context $\vec{u}, \varphi$ would still be true, as long as $\vec{X}=\vec{x}$. 
This condition is perhaps best understood by considering the Suzy-Billy example. It is an attempt to capture the intuition that the reason that Suzy is the cause of the bottle shattering, and not Billy, is that Suzy's rock actually hit the bottle and Billy's didn't. If there is a variable in the model that represents whether Billy's rock hits the bottle, then for Billy's throw to be a cause of the bottle shattering, the bottle would have to shatter (that will be the $\varphi$ in AC2(b)) if Billy throws $(X=x)$ and Suzy does not $(W=w)$ even if, as is actually the case in the real world, Billy's rock does not hit the bottle $\left(Z=z^{*}\right)$. This should become clearer when we analyze this example more formally, in Example 3.3. Before considering this example, I start with a simpler example, just to give the intuitions.

Example 3.2: For the forest-fire example, first consider the context where $U=(1,1,1)$, so that the lightning strikes, the arsonist drops the match, and either one suffices to start the fire. both the dropped match and the lightning are causes of the forest fire in this context. Here is the argument for lightning (the argument for the dropped match is symmetric).

Let $M$ be the causal model for the forest fire described earlier, where the endogenous variables are $L, M D$, and $F F$, and $U$ is the only exogenous variable. In Definition $3.1, \vec{X}=\vec{x}$ is $L=1$ and $\varphi$ is $F F=1$. Clearly $(M,(1,1,1)) \models F F=1$ and $(M,(1,1,1)) \models L=1$; in the context $(1,1,1)$, the lightning strikes and the forest burns down. Thus, AC1 is satisfied. AC3 is trivially satisfied, since $\vec{X}$ consists of only one element, $L$, so must be minimal. For AC2, let $\vec{Z}=\{L, F F\}, \vec{W}=\{M D\}, x^{\prime}=0$, and $w=0$. Clearly, $(M,(1,1,1)) \models$ $[L \leftarrow 0, M D \leftarrow 0](F F \neq 1)$; if the lightning does not strike and the match is not dropped, the forest does not burn down, so AC2(a) is satisfied. To see the effect of the lightning, we must consider the contingency where the match is not dropped; the definition allows us to do that by setting $M D$ to 0 . (Note that setting $L$ and $M D$ to 0 overrides the effects of $U$; this is critical.) Moreover, $(M,(1,1,1)) \models[L \leftarrow 1, M D \leftarrow 0](F F=1)$; if the lightning strikes, then the forest burns down even if the lit match is not dropped, so AC2(b) is satisfied. (Note that since $\vec{Z}=\{L, F F\}$, the only subsets of $\vec{Z}-\vec{X}$ are the empty set and the singleton set consisting of just $F F$.)

The lightning and the dropped match are also causes of the forest fire in the context $(1,1,2)$, where both the lightning and the match are needed to start the fire. Again, I just present the argument for the lightning here. As before, both AC1 and AC3 are trivially satisfied. For AC2, again take $\vec{Z}=\{L, F F\}, \vec{W}=\{M D\}$, and $x^{\prime}=0$, but now let $w=1$. We have that

$$
\begin{gathered}
(M,(1,1,2))=[L \leftarrow 0, M D \leftarrow 1](F F \neq 1) \text { and } \\
(M,(1,1,2)) \models[L \leftarrow 1, M D \leftarrow 1](F F=1),
\end{gathered}
$$

so $\mathrm{AC} 2(\mathrm{a})$ and $\mathrm{AC} 2(\mathrm{~b})$ are satisfied.

As this example shows, causes are not unique; there may be more than one cause of a given outcome. Moreover, both the lightning and the dropped match are causes both in the case where either one suffices to start the fire and in the case where both are needed. As we shall see, the notion of responsibility distinguishes these two situations. Finally, it is worth noting that the lightning is not the cause in either the context $(1,0,2)$ or the context $(1,1,0)$. In the 
first case, $\mathrm{AC} 1$ is violated. If both the lightning and the match are needed to cause the fire, then there is no fire if the match is not dropped. In the second case, there is a fire but, intuitively, it arises spontaneously; neither the lightning nor the dropped match are needed. Here AC2(a) is violated; there is no setting of $L$ and $M D$ that will result in no forest fire.

Example 3.3: Now let us consider the Suzy-Billy example. ${ }^{5}$ We get the desired result-that Suzy's throw is a cause, but Billy's is not-but only if we model the story appropriately. Consider first a coarse causal model, with three endogenous variables:

- ST for "Suzy throws", with values 0 (Suzy does not throw) and 1 (she does);

- BT for "Billy throws", with values 0 (he doesn't) and 1 (he does);

- $B S$ for “bottle shatters', with values 0 (it doesn't shatter) and 1 (it does).

(I omit the exogenous variable here; it determines whether Billy and Suzy throw.) Take the formula for $B S$ to be such that the bottle shatters if either Billy or Suzy throw; that is $B S=$ $B T \vee S T$. (I am implicitly assuming that Suzy and Billy never miss if they throw.) BT and $S T$ play symmetric roles in this model; there is nothing to distinguish them. Not surprisingly, both Billy's throw and Suzy's throw are classified as causes of the bottle shattering in this model. The argument is essentially identical to the forest-fire example in the case that $U=(1,1,1)$, where either the lightning or the dropped match is enough to start the fire.

The trouble with this model is that it cannot distinguish the case where both rocks hit the bottle simultaneously (in which case it would be reasonable to say that both $S T=1$ and $B T=1$ are causes of $B S=1$ ) from the case where Suzy's rock hits first. The model has to be refined to express this distinction. One way is to invoke a dynamic model [Pearl 2000, p. 326]. This model is discussed by HP. A perhaps simpler way to gain expressiveness is to allow $B S$ to be three valued, with values 0 (the bottle doesn't shatter), 1 (it shatters as a result of being hit by Suzy's rock), and 2 (it shatters as a result of being hit by Billy's rock). I leave it to the reader to check that $S T=1$ is a cause of $B S=1$, but $B T=1$ is not (if Suzy doesn't thrown but Billy does, then we would have $B S=2$ ). Thus, to some extent, this solves our problem. But it borders on cheating; the answer is almost programmed into the model by invoking the relation "as a result of", which requires the identification of the actual cause.

A more useful choice is to add two new random variables to the model:

- $B H$ for "Billy's rock hits the (intact) bottle", with values 0 (it doesn't) and 1 (it does); and

- $S H$ for "Suzy's rock hits the bottle", again with values 0 and 1.

Now it is the case that, in the context where both Billy and Suzy throw, $S T=1$ is a cause of $B S=1$, but $B T=1$ is not. To see that $S T=1$ is a cause, note that, as usual, it is immediate

\footnotetext{
${ }^{5}$ The discussion of this and the following example is taken almost verbatim from HP.
} 
that $\mathrm{AC} 1$ and $\mathrm{AC} 3$ hold. For AC2, choose $\vec{Z}=\{S T, S H, B H\}, \vec{W}=\{B T\}$, and $w=0$. When $B T$ is set to $0, B S$ tracks $S T$ : if Suzy throws, the bottle shatters and if she doesn't throw, the bottle does not shatter. To see that $B T=1$ is not a cause of $B S=1$, we must check that there is no partition $\vec{Z} \cup \vec{W}$ of the endogenous variables that satisfies AC2. Attempting the symmetric choice with $\vec{Z}=\{B T, B H, S H\}, \vec{W}=\{S T\}$, and $w=0$ violates AC2(b). To see this, take $\vec{Z}^{\prime}=\{B H\}$. In the context where Suzy and Billy both throw, $B H=0$. If $B H$ is set to 0 , the bottle does not shatter if Billy throws and Suzy does not. It is precisely because, in this context, Suzy's throw hits the bottle and Billy's does not that we declare Suzy's throw to be the cause of the bottle shattering. AC2(b) captures that intuition by allowing us to consider the contingency where $B H=0$, despite the fact that Billy throws. I leave it to the reader to check that no other partition of the endogenous variables satisfies AC2 either.

This example emphasizes an important moral. If we want to argue in a case of preemption that $X=x$ is the cause of $\varphi$ rather than $Y=y$, then there must be a random variable $(B H$ in this case) that takes on different values depending on whether $X=x$ or $Y=y$ is the actual cause. If the model does not contain such a variable, then it will not be possible to determine which one is in fact the cause. This is certainly consistent with intuition and the way we present evidence. If we want to argue (say, in a court of law) that it was $A$ 's shot that killed $C$ rather than $B$ 's, then we present evidence such as the bullet entering $C$ from the left side (rather than the right side, which is how it would have entered had $B$ 's shot been the lethal one). The side from which the shot entered is the relevant random variable in this case. Note that the random variable may involve temporal evidence (if $Y$ 's shot had been the lethal one, the death would have occurred a few seconds later), but it certainly does not have to.

Example 3.4: Can not performing an action be (part of) a cause? Consider the following story, also taken from (an early version of) [Hall 2004]:

Billy, having stayed out in the cold too long throwing rocks, contracts a serious but nonfatal disease. He is hospitalized and treated on Monday, so is fine Tuesday morning.

But now suppose the doctor does not treat Billy on Monday. Is the doctor's omission to treat Billy a cause of Billy's being sick on Tuesday? It seems that it should be, and indeed it is according to our analysis. Suppose that $\vec{u}$ is the context where, among other things, Billy is sick on Monday and the situation is such that the doctor forgets to administer the medication Monday. It seems reasonable that the model should have two random variables:

- $M T$ for "Monday treatment", with values 0 (the doctor does not treat Billy on Monday) and 1 (he does); and

- $B M C$ for "Billy's medical condition", with values 0 (recovered) and 1 (still sick).

Sure enough, in the obvious causal model, $M T=0$ is a cause of $B M C=1$. 
This may seem somewhat disconcerting at first. Suppose there are 100 doctors in the hospital. Although only one of them was assigned to Billy (and he forgot to give medication), in principle, any of the other 99 doctors could have given Billy his medication. Is the fact that they didn't give him the medication also part of the cause of him still being sick on Tuesday?

In the causal model above, the other doctors' failure to give Billy his medication is not a cause, since the model has no random variables to model the other doctors' actions, just as there was no random variable in the causal model of Example 3.2 to model the presence of oxygen. Their lack of action is part of the context. We factor it out because (quite reasonably) we want to focus on the actions of Billy's doctor. If we had included endogenous random variables corresponding to the other doctors, then they too would be causes of Billy's being sick on Tuesday. The more refined definition of causality given in the next section provides a way of avoiding this problem even if the model includes endogenous variables for the other doctors.

With this background, I continue with Hall's modification of the original story.

Suppose that Monday's doctor is reliable, and administers the medicine first thing in the morning, so that Billy is fully recovered by Tuesday afternoon. Tuesday's doctor is also reliable, and would have treated Billy if Monday's doctor had failed to. ... And let us add a twist: one dose of medication is harmless, but two doses are lethal.

Is the fact that Tuesday's doctor did not treat Billy the cause of him being alive (and recovered) on Wednesday morning?

The causal model for this story is straightforward. There are three random variables:

- MT for Monday's treatment (1 if Billy was treated Monday; 0 otherwise);

- TT for Tuesday's treatment (1 if Billy was treated Tuesday; 0 otherwise); and

- $B M C$ for Billy's medical condition (0 if Billy is fine both Tuesday morning and Wednesday morning; 1 if Billy is sick Tuesday morning, fine Wednesday morning; 2 if Billy is sick both Tuesday and Wednesday morning; 3 if Billy is fine Tuesday morning and dead Wednesday morning).

We can then describe Billy's condition as a function of the four possible combinations of treatment/nontreatment on Monday and Tuesday. I omit the obvious structural equations corresponding to this discussion.

In this causal model, it is true that $M T=1$ is a cause of $B M C=0$, as we would expectbecause Billy is treated Monday, he is not treated on Tuesday morning, and thus recovers Wednesday morning. $M T=1$ is also a cause of $T T=0$, as we would expect, and $T T=0$ is a cause of Billy's being alive ( $B M C=0 \vee B M C=1 \vee B M C=2)$. However, $M T=1$ is $n o t$ a cause of Billy's being alive. It fails condition AC2(a): setting $M T=0$ still leads to Billy's 
being alive (with $W=\emptyset$ ). Note that it would not help to take $\vec{W}=\{T T\}$. For if $T T=0$, then Billy is alive no matter what $M T$ is, while if $T T=1$, then Billy is dead when $M T$ has its original value, so $\mathrm{AC} 2(\mathrm{~b})$ is violated (with $\vec{Z}^{\prime}=\emptyset$ ).

This shows that causality is not transitive, according to our definitions. Although $M T=1$ is a cause of $T T=0$ and $T T=0$ is a cause of $B M C=0 \vee B M C=1 \vee B M C=2, M T=1$ is not a cause of $B M C=0 \vee B M C=1 \vee B M C=2$. Nor is causality closed under right weakening: $M T=1$ is a cause of $B M C=0$, which logically implies $B M C=0 \vee B M C=$ $1 \vee B M C=2$, which is not caused by $M T=1$.

This distinguishes the HP definition from that of Lewis [2000], which builds in transitivity and implicitly assumes right weakening.

\section{Dealing with normality and typicality}

While the definition of causality given in Definition 3.1 works well in many cases, it does not always deliver answers that agree with (most people's) intuition. Consider the following example, taken from Hitchcock [2007], based on an example due to Hiddleston [2005].

Example 4.1: Assassin is in possession of a lethal poison, but has a last-minute change of heart and refrains from putting it in Victim's coffee. Bodyguard puts antidote in the coffee, which would have neutralized the poison had there been any. Victim drinks the coffee and survives. Is Bodyguard's putting in the antidote a cause of Victim surviving? According to the preliminary HP definition, it is. For in the contingency where Assassin puts in the poison, Victim survives iff Bodyguard puts in the antidote.

Example 4.1 illustrates an even deeper problem with Definition 3.1. Note that the structural equations for Example 4.1 isomorphic to those in Example 3.2, provided that we interpret the variables appropriately. Specifically, take the endogenous variables in Example 4.1 to be $A$ (for "assassin does not put in poison"), $B$ (for "bodyguard puts in antidote"), and $V S$ (for "victim survives"). Then $A, B$, and $V S$ satisfy exactly the same equations as $L, M D$, and $F F$, respectively. That means that any definition that just depends on the structural equations is bound to give the same answers in these two examples. (A similar example illustrating the same phenomenon is given by Hall [2007].) This suggests that there must be more to causality than just the structural equations. And, indeed, the final HP definition of causality allows certain contingencies to be labeled as "unreasonable" or "too far-fetched"; these contingencies are then not considered in AC2.

As was pointed out in [Halpern 2008], there are problems with the HP account. Halpern [2008] gives an alternative account, which is further refined and developed by Halpern and Hitchcock [2013]. I discuss the latter account here. This account builds on the assumption that the agent has, in addition to a theory of causality (as modeled by the structural equations), a theory of "normality" or "typicality". (The need to consider normality was also stressed by Hitchcock [2007] and Hall [2007], and further explored by Hitchcock and Knobe [2009].) 
This theory would include statements like "typically, people do not put poison in coffee" and "typically doctors do not treat patients to whom they are not assigned".

As a first step to formalizing the notion of normality, take a world to be an assignment of values to all the random variables. Thus, a world in the forest-fire example might be one where $U=(1,1,1), M D=1, L=0$, and $F F=0$; the match is dropped, there is no lightning, and no forest fire (despite the fact that either lightning or a dropped match should be enough for there to be a forest fire). Intuitively, a world is a complete description of a situation given the language at our disposal (the random variables). We want to be able to talk about one world being more normal or typical than another. There are many ways of doing this (see [Friedman and Halpern 2001] and the references therein); for definiteness, Halpern and Hitchcock used a partial preorder $\succeq$ on worlds; $s \succeq s^{\prime}$ means that world $s$ is at least as normal as world $s^{\prime}$. The fact that $\succeq$ is a partial preorder means that it is reflexive (for all worlds $s$, we have $s \succeq s$, so $s$ is at least as normal as itself) and transitive (if $s$ is at least as normal as $s^{\prime}$ and $s^{\prime}$ is at least as normal as $s^{\prime \prime}$, then $s$ is at least as normal as $\left.s^{\prime \prime}\right){ }^{6}$ A partial preorder allows the worlds to be incomparable: it may be the case that neither $s \succeq s^{\prime}$ nor $s^{\prime} \succeq s$ holds. ${ }^{7}$

An extended causal model is a tuple $M=(\mathcal{S}, \mathcal{F}, \succeq)$, where $(\mathcal{S}, \mathcal{F})$ is a causal model, and $\succeq$ is a partial preorder on worlds. Note that in an acyclic extended causal model, a context $\vec{u}$ determines a world, denoted $s_{\vec{u}}$. We can now modify Definition 3.1 slightly to take the relative normality of worlds (as given by $\succeq$ ) into account by taking $\vec{X}=\vec{x}$ to be a cause of $\varphi$ in an extended model $M$ and context $\vec{u}$ if $\vec{X}=\vec{x}$ is a cause of $\varphi$ according to Definition 3.1, except that in AC2(a), there must be a world $s$ such that $s \succeq s_{\vec{u}}$ and $s_{\vec{X}=\vec{x}^{\prime}, \vec{W}=\vec{w}, \vec{u}} \succeq s_{\vec{u}}$, where $s_{\vec{X}=\vec{x}^{\prime}, \vec{W}=\vec{w}, \vec{u}}$ is the world that results by setting $\vec{X}$ to $\vec{x}^{\prime}$ and $\vec{W}$ to $\vec{w}$ in context $\vec{u}$. This modification can be viewed as a formalization of Kahneman and Miller's [1986] observation that "an event is more likely to be undone by altering exceptional than routine aspects of the causal chain that led to it". In AC2(a), only worlds $s$ that are at least as normal as the actual world $s_{\vec{u}}$ are considered, so indeed, it is "exceptional aspects" that are being altered rather than "routine aspects" (since altering routine aspects would presumably lead to a less normal world, while altering exceptional aspects would lead to a more normal world).

This definition deals well with all the problematic examples in the literature. Consider Example 4.1. In the actual world, $A=1, B=1$, and $V S=1$ : the assassin does not put in poison, the bodyguard puts in the antidote, and the victim survives. The witness for $B=1$ to be an actual cause of $V S=1$ is the world where $A=0, B=0$, and $V S=0$. If we make the assumption that $A$ typically takes the value 1 and $B$ typically take the value $0,{ }^{8}$ we get a normality ordering in which the two worlds $(A=1, B=1, V S=1)$ and $(A=0, B=0, V S=0)$ are incomparable. Since the unique witness for $B=1$ to be an

\footnotetext{
${ }^{6}$ If $\succeq$ were a partial order rather than just a partial preorder, it would satisfy an additional assumption, antisymmetry: $s \succeq s^{\prime}$ and $s^{\prime} \succeq s$ implies $s=s^{\prime}$. This is an assumption that I do not make here.

${ }^{7}$ This makes partial preorders more general than the ranking functions [Spohn 1988] used in [Halpern 2008] to capture the relative normality of worlds. This extra generality turns out to be useful to capture a number of examples.

${ }^{8}$ Although it may be atypical for an assassin to poison a victim's drink, the action is morally wrong and unusual from the victim's perspective, both of which would tend to make it atypical.
} 
actual cause of $V S=1$ is incomparable with the actual world, our modified definition rules that $B=1$ is not an actual cause of $V S=1$.

Now consider the first variant of Example 3.4 where there are 100 doctors, none of whom treat Billy. Intuitively, we want to call Billy's doctor the cause of Billy's still being sick, since he did not treat Billy, but we do not want to call the other 99 doctors causes, despite the fact that they did not treat Billy either. The way this was dealt with in Example 3.4 was by not having variables in the model corresponding to the other 99 doctors. By having a theory of normality in the model, we can deal with this issue even in a model that includes variables for all the other doctors. Consider an extended causal model with variables $A_{1}, \ldots, A_{100}$ and $M T_{1}, \ldots, M T_{100}$ in the causal model, where $A_{i}=1$ if doctor $i$ is assigned to treat Billy and $A_{i}=0$ if he is not, and $M T_{i}=1$ if doctor $i$ actually treats Billy on Monday, and $M T_{i}=0$ if he does not. Further assume that, typically, no doctor is assigned to a given patient; if doctor $i$ is not assigned to treat Billy, then typically doctor $i$ does not treat Billy; and if doctor $i$ is assigned to Billy, then typically doctor $i$ treats Billy. This can be captured by making the world where no doctor is assigned to Billy and no doctor treats him more normal than the 100 worlds where exactly one doctor is assigned to Billy, and that doctor treats him, which in turn are more normal than the 100 worlds where exactly one doctor is assigned to Billy and no one treats him have rank 2, which in turn are more normal than the $100 \times 99$ worlds where exactly one doctor is assigned to Billy but some other doctor treats him. (The relative normality of the remaining worlds is irrelevant.) In this extended model, in the context where doctor $i$ is assigned to Billy but no one treats him, $i$ is the cause of Billy's sickness (the world where $i$ treats Billy is more normal than the world where $i$ is assigned to Billy but no one treats him), but no other doctor is a cause of Billy's sickness. Moreover, in the context where $i$ is assigned to Billy and treats him, then $i$ is the cause of Billy's recovery (for AC2(a), consider the world where no doctor is assigned to Billy and none treat him).

The use of normality also gives some insight into the distinction between exogenous and endogenous variables (and the notion of condition vs. causality in the legal literature). I earlier said that, typically, we would take the presence of oxygen to be exogenous (or would not even bother having a variable to describe it). One way of understanding the logic behind this is that the presence of oxygen is so much more normal than its absence that there is no point in making it endogenous (and thus considering changing it). We can understand the distinction between conditions and causes the same way. Conditions represent values of variables that are much more normal than all other possible values; (potential) causes are quite often those things whose actual value is somewhat abnormal or atypical.

Adding a representation of normality to the model has various other advantages, as discussed by Halpern and Hitchcock [2013]. In particular, it allows us to capture instances of the legal doctrine of intervening causes. In the law, it is held that one is not causally responsible for some outcome when one's action led to that outcome only via the intervention of a later agent's deliberate action, or some very improbable event. For example, if Anne negligently spills gasoline, and Bob carelessly throws a cigarette in the spill, then Anne's action is a cause of the fire. But if Bob maliciously throws a cigarette in the gas, then Anne is not considered a 
cause [Hart and Honoré 1985]. ${ }^{9}$ This example can be captured using an extended causal model (see [Halpern and Hitchcock 2013] for details).

Although the addition of normality to the causation picture gives a great deal of added modeling power, it raises the worry that it gives the modeler too much flexibility. After all, the modeler can now render any claim that $A$ is an actual cause of $B$ false, simply by choosing a normality order that assigns the actual world $s_{\vec{u}}$ a lower rank than any world $s$ needed to satisfy AC2. Thus, the introduction of normality exacerbates the problem of motivating and defending a particular choice of model. Fortunately, the literature on the psychology of counterfactual reasoning and causal judgment goes some way towards enumerating the sorts of factors that constitute normality. (See, e.g., [Alicke 1992; Cushman 2009; Cushman, Knobe, and Sinnott-Armstrong 2008; Hitchcock and Knobe 2009; Kahneman and Miller 1986; Knobe and Fraser 2008; Kahneman and Tversky 1982; Mandel, Hilton, and Catellani 1985; Roese 1997].) These include statistical frequency (things that occur more frequently are more normal), moral judgments (actions that follow moral precepts are more normal), and agreed-upon conventions (following a convention is more normal that not); see [Halpern and Hitchcock 2010] for more discussion.

In particular, the law suggests a variety of principles for determining the norms that are used in the evaluation of actual causation. In criminal law, norms are determined by direct legislation. For example, if there are legal standards for the strength of seat belts in an automobile, a seat belt that did not meet this standard could be judged a cause of a traffic fatality. By way of contrast, if a seat belt complied with the legal standard, but nonetheless broke because of the extreme forces it was subjected to during a particular accident, the fatality would be blamed on the circumstances of the accident, rather than the seat belt. In such a case, the manufacturers of the seat belt would not be guilty of criminal negligence. In contract law, compliance with the terms of a contract has the force of a norm. In tort law, actions are often judged against the standard of "the reasonable person". For instance, if a bystander was harmed when a pedestrian who was legally crossing the street suddenly jumped out of the way of an oncoming car, the pedestrian would not be held liable for damages to the bystander, since he acted as the hypothetical "reasonable person" would have done in similar circumstances. (See, e.g., [Hart and Honoré 1985, pp. 142ff.] for discussion.) There are also a number of circumstances in which deliberate malicious acts of third parties are considered to be "abnormal" interventions, and affect the assessment of causation. (See, e.g., [Hart and Honoré 1985, pp. 68ff.].)

\section{Responsibility and blame}

The HP definition of causality treats causality as an all-or-nothing concept (as do all the other definitions of causality in the literature that I am aware of). While we can talk about the probability that $A$ is a cause of $B$ (by putting a probability on contexts), we cannot talk about the degree of causality. This means that we cannot make some distinctions that seem intuitively

\footnotetext{
${ }^{9}$ This example is based on the facts of Watson v. Kentucky and Indiana Bridge and Railroad [1910].
} 
significant. For example, as we observed earlier, there seems to be a difference in the degree of responsibility of a voter for a victory in an 11-0 election and a 6-5 election. As Chockler and Halpern showed, one of the advantages of the HP definition is that it provides a straightforward way of defining refinements of the notion of causality that let us capture important intuitions regarding degree of responsibility and blame. The discussion in this section closely follows that in [Chockler and Halpern 2004].

\subsection{Responsibility}

The idea behind the definition of degree of responsibility is straightforward: If $A$ is not a cause of $B$ then the degree of responsibility of $A$ for $B$ is 0 . If $A$ is a cause of $B$, then the degree of responsibility of $A$ for $B$ is $1 /(N+1)$, where $N$ is the minimal number of changes that have to be made to obtain a contingency where $B$ counterfactually depends on $A$. In the case of the $11-0$ vote, the degree of responsibility of any voter for the victory is $1 / 6$, since 5 changes have to be made before a vote is critical. If the vote were 1001-0, the degree of responsibility of any voter would be $1 / 501$. On the other hand, if the vote is $6-5$, then the degree of responsibility of each of the 6 voters in favor of the outcome is 1; each of them is critical. (Of course, the 5 voters who were not in favor have responsibility 0 for the outcome.)

Thus, the degree of responsibility of $A$ for $B$ is a number between 0 and 1 . It is a refinement of the notion of causality. It is 0 if and only if $A$ is not a cause of $B$. If $A$ is a cause of $B$, then the degree of responsibility is positive. Despite being a number between 0 and 1 , as the voting examples make clear, degree of responsibility does not act like probability at all.

Here is the formal definition of degree of responsibility, from [Chockler and Halpern 2004] (modified to be appropriate for extended causal models rather than just causal models).

Definition 5.1: The degree of responsibility of $\vec{X}=\vec{x}$ for $\varphi$ in $(M, \vec{u})$, denoted $d r((M, \vec{u}),(\vec{X}=$ $\vec{x}), \varphi)$, is 0 if $\vec{X}=\vec{x}$ is not a cause of $\varphi$ in $(M, \vec{u})$; it is $1 /(k+1)$ if $\vec{X}=\vec{x}$ is a cause of $\varphi$ in $(M, \vec{u})$ and there exists a partition $(\vec{Z}, \vec{W})$ and a setting $\left(\vec{x}^{\prime}, \vec{w}\right)$ that determines a world at least as normal as $s_{\vec{u}}$ for which AC2 holds such that (a) $k$ variables in $\vec{W}$ have different values in $\vec{w}$ than they do in the context $\vec{u}$ and (b) there is no partition $\left(\vec{Z}^{\prime}, \vec{W}^{\prime}\right)$ and setting $\left(\vec{x}^{\prime \prime}, \vec{w}^{\prime}\right)$ satisfying AC2 such that only $k^{\prime}<k$ variables have different values in $\vec{w}^{\prime}$ than they do in $\vec{u}$.

It should be clear that the degree of responsibility for the voting examples is indeed $1 / 6$ in the case of an 11-0 victory and 1 in the case of a 6-5 victory. It is easy to see that in the context $(1,1,1)$ in the forest-fire example, where the lightning strikes, the arsonist drops the match, and either one suffices for a fire, the lightning and the arsonist each have degree of responsibility $1 / 2$ for the fire (assuming that the setting where the arsonist doesn't drop the match and where the lightning does not strike are both allowable, which seems reasonable). On the other hand, in the context $(1,1,2)$, where both are needed for the fire, then the lightning and the arsonist each have degree of responsibility 1. Finally, in the Suzy-Billy example, since Billy is not a cause, Billy has degree of responsibility 0. Suzy's degree of responsibility depends on which settings 
are allowable in the extended causal model. If we take $\vec{W}$ to be $\{B T, B H\}$, and keep both variables at their actual setting in the context, so that $B T=1$ and $B H=0$, then Suzy's throw becomes critical; if she throws, the bottle shatters, and if she does not throw, the bottle does not shatter (since $B H=0)$. On the other hand, if the setting $(S T=0, B T=1, B H=0)$ is not allowable in the extended causal model (on the grounds that it requires Billy's throw to miss), but the arguably more reasonable settings $(S T=0, B T=0, B H=0)$ and $(S T=1, B T=$ $1, B H=0)$ where Billy does not throw are allowable, then Suzy's degree of responsibility is $1 / 2$, since we must consider the contingency where Billy does not throw. Thus, by using an appropriate extended causal model, an important intuition can be captured.

While the notion of degree of responsibility seems important, and defining it in terms of the number of changes needed to make a variable critical captures some reasonable intuitions, it is admittedly a naive definition. In some contexts, it seems to come closer to our intuitions to have the degree of responsibility of $\vec{X}=\vec{x}$ decrease exponentially with the number of changes needed to make $\vec{X}=\vec{x}$ critical, so that the degree of responsibility would be, say, $1 / 2^{k}$ rather than $1 /(k+1)$ if $k$ changes are needed. ${ }^{10}$ It may also be appropriate to assign weights to variables. To understand the intuition for this, consider a variant of the voting example, where there are two voters, and each one controls a block of votes: voter 1 controls 8 votes and voter 2 controls 3 votes. Moreover, each voter must cast all his votes for one candidate; votes cannot be split. (This is like voting in the U.S. Electoral College. If we consider a "voter" as representing a state in the Electoral College, for all states besides Nebraska and Maine, vote splitting is not allowed; the winner of the popular vote in the state gets all the state's electoral votes.) If both voters vote for Mr. B, then only voter 1 is the cause of Mr. B's victory, and thus has degree of responsibility 1 . On the other hand, if vote splitting is allowed (so that voter 1 can be represented by a random variable that takes on values $0, \ldots, 8$, while voter 2 can be represented by a random variable that takes on values $0, \ldots, 3)$, then both voter 1 and voter 2 are causes of Mr. B's victory; however, voter 1 has degree of responsibility 1 (his vote is clearly critical), while voter 2 has degree of responsibility $1 / 2$ (since voter 2 becomes critical if, e.g., voter 1 splits his vote $4-4$ ). Note that, if vote splitting is allowed, voter 2 would continue to have degree of responsibility $1 / 2$ even if he controlled only one vote and voter 1 controlled 1,000 votes.

Some might think that voter 2 should have a lower degree of responsibility, which takes into account the fact that he controls fewer votes. We could capture this by assigning different weights to voters (or, more precisely, to the variables representing voters), and having the definition of degree of responsibility use this weight (rather than implicitly weighting all voters equally). That is, the degree of responsibility of $\vec{X}=\vec{x}$ for $\varphi$ would be 1 over $1+$ the sum of the weights of the variables that need to be changed to make $X=x$ critical. While this would give voter 2 lower responsibility, it requires a modeler to assign a weight to variables. Moreover, it is not so clear that weighting the variables captures all our intuitions here. For example, suppose that, as before, voter 1 controls 8 votes, voter 2 controls 3 votes, but now there is a third voter that controls 10 votes. If voters 1 and 2 vote for Mr. B and voter 3 votes for Mr. G,

\footnotetext{
${ }^{10}$ I thank Denis Hilton for this suggestion.
} 
many would agree that it is reasonable to assign both voters 1 and 2 degree of responsibility 1 for the outcome.

An alternative that would not require any additional information from the modeler would be to have the degree of responsibility of $\vec{X}=\vec{x}$ for $\varphi$ depend on how many different changes make $\vec{X}=\vec{x}$ critical. For example, if voter 1 controls 8 votes and voter 2 controls 3 , under this approach, voter 1 still has degree of responsibility 1 , because all changes to voter 2's votes make voter 1 critical. On the other hand, only three of the eight possible changes to voter 1 's vote (making the split 3-5, 4-4, or 5-3) make voter 2 critical. Thus, voter 2's degree of responsibility would be $3 / 8$. I have not explored the implications of this modification of the definition. While in the rest of the paper I use the definition of degree of responsibility in Definition 5.1, a variant may well be more appropriate in some applications. In particular, while Definition 5.1 was argued to be useful for model checking (an approach to verifying the correctness of computer programs) [Chockler, Halpern, and Kupferman 2008], the variant that assigns weights to variables turns out to be useful in another model-checking context [Chockler, Grumberg, and Yadgar 2008]. Of course, legal applications may have yet different requirements.

Interestingly, there is some evidence that people do use something like the procedure defined here to ascribe responsibility [Gerstenberg and Lagnado 2010], although more recently it has been argued that people take into account not only the number of changes required to make a particular event critical, but how many ways there are to reach a situation where that event is critical [Zultan, Gerstenberg, and Lagnado 2012]. The latter point could be captured in a straightforward way by making a small modification to the definition of responsibility. Again, further experimentation is required to see whether this is the "right" definition for legal applications.

\subsection{Blame}

The definitions of both causality and responsibility are relative to an extended causal model and a context. Thus, they implicitly assume that both are given; there is no uncertainty. Once we have a probability on contexts and causal models, we can talk about the probability of causality and the expected degree of responsibility. The latter notion is called (degree of) blame by Chockler and Halpern [2004].

Obviously, if we add probability to the picture, we must address the question of where the probability is coming from. In some cases it might be objective; that is, it might come from a source with an agreed-upon probability, like a coin toss. Or we may have statistical information that determines the probability. Alternatively, the probability may represent the agent's subjective beliefs. The definitions make sense with either interpretation.

To take a simple example of how probability can be used, suppose that an agent is unsure as to whether the context in the forest-fire example is $(1,0,1),(1,1,1)$, or $(1,1,2)$, and assigns each of these contexts has probability $1 / 3$. (For now let us not worry about where the probability is coming from.) With these probabilities, the probability that the arsonist dropping the match 
is a cause of fire is $2 / 3$ (since it is a cause in context $(1,1,1)$ and $(1,1,2)$, but not in context $(1,0,1)$. The degree of responsibility of the match is 0 in context $(1,0,1), 1$ in the contexts $(1,1,2)$ and $(1,1,2)$, and $1 / 2$ in the context $(1,1,1)$, where either the lightning or the dropped match suffices to cause the forest fire. Thus, the degree of blame (i.e., expected degree of responsibility $)$ is $1 / 2(=0 \times 1 / 3+1 \times 1 / 3+1 / 2 \times 1 / 3)$.

As I said earlier, there are two significant sources of uncertainty for an agent who is contemplating performing an action:

- what the true situation is (i.e., what value the exogenous variables have); for example, a doctor may be uncertain about whether a patient has high blood pressure;

- how the world works; for example, a doctor may be uncertain about the side effects of a given medication.

In the HP framework, the "true situation" is determined by the context; "how the world works" is determined by the equations. All the uncertainty about the equations can be encoded into the context. For example, in modeling the forest fire, I used the context $U$ to describe whether both the match and the lightning are needed for the fire, or whether one of them suffices. But at times it is convenient to simply use two different causal models. For example, we can imagine a causal model for cancer where smoking causes cancer, and another causal model where cancer is unrelated to smoking While we now believe that the first causal model more accurately depicts reality, at one point there was some doubt.

Define a situation to be a pair of the form $(M, \vec{u})$, where $M$ is an extended causal model and $\vec{u}$ is a context. As the discussion above suggests, an agent has uncertainty regarding the true situation. I thus take an agent's uncertainty to be modeled by a pair $(\mathcal{K}, \operatorname{Pr})$, where $\mathcal{K}$ is a set of situations and $\operatorname{Pr}$ is a probability distribution over $\mathcal{K}$. In the forest-fire example, in all the situations, the causal model was the same, but in general this need not be the case. Intuitively, $\mathcal{K}$ describes the situations that the agent considers possible before $\vec{X}$ is set to $\vec{x}$. (Note that the situation $\left(M_{\vec{X} \leftarrow \vec{x}}, \vec{u}\right)$ for $(M, \vec{u}) \in \mathcal{K}$ are those that the agent considers possible after $X$ is set to $x$.) The degree of blame that setting $\vec{X}$ to $\vec{x}$ has for $\varphi$ is then the expected degree of responsibility of $\vec{X}=\vec{x}$ for $\varphi$ in $\left(M_{\vec{X} \leftarrow \vec{x}}, \vec{u}\right)$, taken over the situations $(M, \vec{u}) \in \mathcal{K}$.

Definition 5.2: The degree of blame of $\vec{X}=\vec{x}$ for $\varphi$ relative to epistemic state $(\mathcal{K}, \operatorname{Pr})$, denoted $d b(\mathcal{K}, \operatorname{Pr}, \vec{X} \leftarrow \vec{x}, \varphi)$, is

$$
\sum_{(M, \vec{u}) \in \mathcal{K}} d r\left(\left(M_{\vec{X} \leftarrow \vec{x}}, \vec{u}\right), \vec{X}=\vec{x}, \varphi\right) \operatorname{Pr}((M, \vec{u})) .
$$

In this definition, it is perhaps best to think of $\vec{X}=\vec{x}$ as indicating that some action has been performed. Thus, if we are trying to decide to what extent an agent who performs a particular action is to blame for an outcome, we can take $X$ to be a random variable that indicates whether the action is performed (so that $X=1$ if the action is performed and $X=0$ otherwise) and 
$\varphi$ to be the outcome of interest. To determine the degree of blame attached to $X=x$, we first consider what situations the agent considers possible before the action is performed, and how likely each one of them is (according to the agent). This is given by $(\mathcal{K}, \operatorname{Pr})$. We then consider the agent's degree of responsibility in each model that arises if the action is actually performed. If $(M, u)$ is one of the situations the agent considers possible before performing the action, after performing the action, the situation is described by $M_{X \leftarrow 1}$; we thus consider the degree of responsibility of $X=1$ for the outcome $\varphi$ in the causal model $M_{X \leftarrow 1}$. It may also make sense to put a probability on the situations that arise after the action is performed. I return to this issue below, after considering a few examples.

Example 5.3: Suppose that we are trying to compute the degree of blame of Suzy's throwing the rock for the bottle shattering. Suppose that the only causal model that Suzy considers possible is essentially like the second model in Example 3.3 (with $S H$ and $B H$ ), with some minor modifications: $B T$ can now take on three values, say $0,1,2$; as before, if $B T=0$ then Billy doesn't throw, if $B T=1$, then Billy does throw, and if $B T=2$, then Billy throws extra hard. Assume that the causal model is such that if $B T=1$, then Suzy's rock will hit the bottle first, but if $B T=2$, they will hit simultaneously. Thus, $S H=1$ if $S T=1$, and $B H=1$ if $B T=1$ and $S H=0$ or if $B T=2$. Call this structural model $M$.

At time 0, Suzy considers the following four situations equally likely:

- $\left(M, \vec{u}_{1}\right)$, where $\vec{u}_{1}$ is such that Billy already threw at time 0 (and hence the bottle is shattered);

- $\left(M, \vec{u}_{2}\right)$, where the bottle was whole before Suzy's throw, and Billy throws extra hard, so Billy's throw and Suzy's throw hit the bottle simultaneously (this essentially gives the first model in Example 3.3);

- $\left(M, \vec{u}_{3}\right)$, where the bottle was whole before Suzy's throw, and Suzy's throw hit before Billy's throw (this essentially gives the second model in Example 3.3); and

- $\left(M, \vec{u}_{4}\right)$, where the bottle was whole before Suzy's throw, and Billy did not throw.

The bottle is already shattered in $\left(M, \vec{u}_{1}\right)$ before Suzy's action, so Suzy's throw is not a cause of the bottle shattering, and her degree of responsibility for the shattered bottle is 0. Suzy's degree of responsibility in $\left(M, \vec{u}_{2}\right)$ is $1 / 2$-both Suzy and Billy are equally responsible. In $\left(M, \vec{u}_{4}\right)$, Suzy's degree of responsibility is clearly 1; Billy's not throwing makes Suzy's throw critical. As discussed earlier, Suzy's degree of responsibility in $\left(M, \vec{u}_{3}\right)$ depends on the ranking function $\kappa$. If the setting $(S T=0, B T=1, B H=0)$ is allowable, then her degree of responsibility is 1 ; otherwise it is $1 / 2$. In the former case, the degree of blame is $\frac{1}{4} \cdot \frac{1}{2}+\frac{1}{4} \cdot 1+\frac{1}{4} \cdot 1=\frac{5}{8}$; in the latter case, it is $\frac{1}{4} \cdot \frac{1}{2}+\frac{1}{4} \cdot \frac{1}{2}+\frac{1}{4} \cdot 1=\frac{1}{2}$.

If instead we consider Suzy's probability on situations after the rock is thrown and Suzy observes what happens, then she knows the outcome with probability 1 . Thus, her degree of blame is exactly her degree of responsibility. If Suzy is considering whether to throw the rock 
and wants to consider how much she will be to blame if the bottle shatters, it seems more appropriate to use her prior probability on situations. If she is considering her degree of blame given what has happened, then it makes more sense to use the posterior probability.

Example 5.4: Consider a firing squad with ten excellent marksmen. Suppose that marksman 1 knows that exactly one marksman has a live bullet in his rifle, and that all the marksmen will shoot. Thus, he considers 10 situations possible, depending on who has the bullet. Let $p_{i}$ be some marksman 1's prior probability that marksman $i$ has the live bullet. In situation $i$, marksman $i$ is the cause of death and has degree of responsibility 1 ; in all other situations, marksman $i$ is not the cause of death and has degree of responsibility 0 . Thus, the probability that marksman $i$ is the cause of death is $p_{i}$, and marksman $i$ 's degree of blame is also $p_{i}$. Note that if marksman 1 mistakenly believes that he has the bullet (and thus takes $p_{1}=1$ ) when in fact it is marksman 2, then it is possible for the degree of blame of marksman 1 (according to marksman 1) to be 1 , while in fact the degree of responsibility of marksman 1 is 0 . This shows that degree of blame is a subjective notion, depending on an agent's subjective probability.

If the marksman never actually discovers which bullet was live, then his prior probability is the same as the posterior probability; it does not matter which is used to compute the degree of blame. If he discovers which bullet is live, then his degree of blame will be equal to the degree of responsibility. Finally, if he is given only partial information about which bullet is live, then an appropriate degree of blame based on his posterior probability can be computed.

What an agent's believes is quite relevant to the law. I briefly point out two issues here:

- In some cases, we are interested not in what agents actually believe, but in what they should have believed (had they taken the trouble to get relevant information). The definition of blame does not change; the definition is agnostic as to what epistemic state should be considered. Considering the actual epistemic state is relevant when considering intent; what the epistemic state should have been may be more appropriate in assessing liability. Consider, for example, a patient who dies as a result of being treated by a doctor with a particular drug. Assume that the patient died due to the drug's adverse side effects on people with high blood pressure and, for simplicity, that this was the only cause of death. Suppose that the doctor was not aware of the drug's adverse side effects. (Formally, this means that he does not consider possible a situation with a causal model where taking the drug causes death.) Then, relative to the doctor's actual epistemic state, the doctor's degree of blame will be 0 . However, a lawyer might argue in court that the doctor should have known that treatment had adverse side effects for patients with high blood pressure (because this is well documented in the literature) and thus should have checked the patient's blood pressure. If the doctor had performed this test, he would have known that the patient had high blood pressure. With respect to the resulting epistemic state, the doctor's degree of blame for the death is quite high. Of course, the lawyer's job is to convince the court that the latter epistemic state is the appropriate one to consider when assigning degree of blame. 
- Up to now I have considered the agent's prior probability. But we can also consider the posterior probability. The doctor's epistemic state after a patient's death is likely to be quite different from her epistemic state before the patient's death. She may still consider it possible that the patient died for reasons other than the treatment, but will consider causal structures where the treatment was a cause of death more likely. Thus, the doctor will likely have higher degree of blame relative to her epistemic state after the treatment.

Interestingly, all three epistemic states - the epistemic state that an agent actually has before performing an action, the epistemic state that the agent should have had before performing the action, and the epistemic state after performing the action-have been considered relevant to determining responsibility according to different legal theories [Hart and Honoré 1985, p. 482]. Of course, it may not be so easy to discover what agents know or believe. Corporations and defendants often try to cover-up what they knew and when they knew it by deleting emails, not preserving documents, and so on. Nevertheless, I think it is useful to have definitions that take beliefs into account, and to define notions like blame that depend on beliefs.

Although I have only scratched the surface of these issues here, I hope it is clear that the framework of structural equations and the definitions based on it provide us with some useful tools to address them.

\section{The NESS approach}

As I said, there has been extensive research on causality in both philosophy and law. Halpern and Pearl [2005a] compared the HP approach to other work in the philosophy literature, so I focus here on work in the legal literature. Perhaps the best worked-out approach is the NESS (Necessary Element of a Sufficient Set) test, originally described by Hart and Honoré, and worked out in much greater detail by Wright [1985, 1988, 2001]. Thus, I compare the HP approach to the NESS approach here.

Wright does not provide a mathematical formalization of the NESS test; what I give here is my understanding of it. $A$ is a cause of $B$ according to the NESS test if there exists a set $\mathcal{S}=\left\{A_{1}, \ldots, A_{k}\right\}$ of events, each of which actually occurred, where $A=A_{1}, \mathbf{S}$ is sufficient for $B$, and $\mathbf{S}-\left\{A_{1}\right\}$ is not sufficient for $B$. Thus, $A$ is an element of a sufficient condition for $B$, namely $\mathbf{S}$, and is a necessary element of that set, because any subset of $\left\{A_{1}, \ldots, A_{k}\right\}$ that does not include $A$ is not sufficient for $B .{ }^{11}$

The NESS test, as stated, seems intuitive and simple. Moreover, it deals well with many examples. Consider the forest fire. The lightning and the arsonist are clearly both causes; we can take the set $\mathbf{S}$ to be the singleton set consisting of either lightning or the arsonist dropping a match. Similarly, in Example 3.4, if both Monday's doctor treating Billy and Tuesday's doctor not treating Billy are elements of $\mathrm{S}$, then each of them are causes. However, I believe that

\footnotetext{
${ }^{11}$ The NESS test is much in the spirit of Mackie's [1965] INUS test, according to which $A$ is a cause of $B$ if $A$ is an insufficient but necessary part of a condition which is unnecessary but sufficient for $B$. However, a comparison of the two approaches is beyond the scope of this paper.
} 
the NESS approach has problems with the Suzy-Billy example. These are best pointed out by considering a related example also considered by Wright [1985]. This example shows that, although the NESS test looks quite formal, it lacks a definition of what it means for a set $\mathbf{S}$ of events to be sufficient for $B$ to occur; moreover, such a definition is sorely needed.

Example 6.1: First, suppose that Pamela drinks a cup of tea poisoned by Claire, and then dies. It seems clear that Claire poisoning the tea caused Pamela's death. It seems reasonable in this case to take $\mathbf{S}$ to consist of two events, both of which actually occurred:

- $A_{1}$, Claire poisoned the tea; and

- $A_{2}$, Pamela drank the tea.

Given our understanding of the world, it seems reasonable to say that the $A_{1}$ and $A_{2}$ are sufficient for Pamela's death, but removing $A_{1}$ results in a set that is insufficient.

But now suppose that David shoots Pamela just after she drinks the tea, and she dies instantaneously from the shot (before the poison can take effect). In this case, we would want to say that David's shot is the cause of Pamela's death, not Claire's poisoning. Nevertheless, it would seem that the same argument that makes Claire's poisoning a cause without David's shot would still make Claire's poisoning a cause even without David's shot. The set $\left\{A_{1}, A_{2}\right\}$ still seems sufficient for Pamela's death, while $\left\{A_{2}\right\}$ is not.

Wright [1985] observes the poisoned tea would be a cause of Pamela's death only if Pamela "drank the tea and was alive when the poison took effect". Wright seems to be arguing that $\left\{A_{1}, A_{2}\right\}$ is in fact not sufficient for Pamela's death. We need $A_{3}$ : Pamela was alive when the poison took effect. While I agree that the fact that Pamela was alive when the poison took place is critical for causality, I do not see how it helps in the NESS test, under what seems to me the most obvious definitions of "sufficient". I would argue that $\left\{A_{1}, A_{2}\right\}$ is in fact just as sufficient for death as $\left\{A_{1}, A_{2}, A_{3}\right\}$. For suppose that $A_{1}$ and $A_{2}$ hold. Either Pamela was alive when the poison took effect, or she was not. In the either case, she dies. In the former case, it is due to the poison; in the latter case, it is not.

But it gets worse. While I would argue that $\left\{A_{1}, A_{2}\right\}$ is indeed just as sufficient for death as $\left\{A_{1}, A_{2}, A_{3}\right\}$, it is not clear that $\left\{A_{1}, A_{2}\right\}$ is in fact sufficient. Suppose, for example, that some people are naturally immune to the poison that Claire used, and do not die from it. Pamela is not immune. Then it seems that we need to add a condition $A_{4}$ saying that Pamela is not immune from the poison to get a set sufficient to cause Pamela's death. And why should it stop there? Suppose that the poison has an antidote that, if administered within five minutes of the poison taking effect, will prevent death. Unfortunately, the antidote was not administered to Pamela. Do we have to add this condition to $\mathbf{S}$ to get a sufficient set for Pamela's death? Where does it stop?

Note that in the causal model where the only random variables are "Claire poisoned the tea", "David shot Pamela", "Pamela was alive when the poison took effect", and "Pamela dies" (where the random variable has value 1 or 0 , depending on whether the event happened), 
with the obvious equations, the shot is indeed a cause of death in the HP definition, while the poisoning is not. The argument is almost identical to the Suzy-Billy case. Moreover, adding additional random variables like "Claire is naturally immune" or "the antidote was administered" does not make a difference. However, in general, adding more variables might make a difference. (Recall that, in the Suzy-Billy example, adding the variables $B H$ and $S H$ was important, as is "Pamela was alive when the poison took effect" in this case.) That is why the causal model must make explicit what variables are being considered. $\mathbf{I}$

The issue of what counts as a sufficient cause is further complicated if there is uncertainty about the causal structure.

Example 6.2: Suppose that a doctor gives a patient a new drug to deal with a heart ailment, and then the patient dies. Is the new drug the cause of death? Even ignoring all the issues raised in Example 6.1, it is clear that the answer depends on whether giving the drug is a sufficient condition to cause death (given all the other factors affecting the patient). The NESS test seems to implicitly assume that this is known. For example, Wright [2007] says that the putative cause "must be part of the complete instantiation of the antecedent of the relevant causal law". But the notion of a causal law is undefined, nor is it defined when a causal law is "relevant". By way of contrast, in causal models, the causal laws are encoded by the structural equations.

There is another (less serious) problem with the definition of NESS: which events can go in S. This already arises in the analysis of Example 3.4. Can S include "negative" events like "Tuesday's doctor did not treat Billy". If so, can it also include "Doctor $i$ did not treat Billy" for $i=1, \ldots, 99$, for the other 99 doctors who did not treat Billy? The next example shows that the problem is quite pervasive.

Example 6.3: Wright [2001] considers an example where defendant 1 discharged 15 units of effluent, while defendant 2 discharged 13 units. Suppose that 14 units of effluent are sufficient for injury. It seems clear that defendant 1's discharge is a cause of injury; if he hadn't discharged any effluent, then there would have been no injury. What about defendant 2's discharge? In the HP approach, whether it is a cause depends on the random variables considered and their possible values. Suppose that $D_{i}$ is a random variable representing defendant $i$ 's discharge, for $i=1,2$. If $D_{1}$ can only take values 0 or 15 (i.e., if defendant 1 discharges either nothing or all 15 units), then defendant 2's discharge is not a cause. But if $D_{1}$ can take, for example, every integer value between 0 and 15 , then $D_{2}=13$ is a cause (e.g., under the contingency that $D_{1}=4$ ).

Intuitively, the decision as to whether the causal model should include 4 as a possible value of $D_{1}$ or have 0 and 15 as the only possible values of $D_{1}$ should depend on the options available to defendant 1 . If all he can do is to press a switch that determines whether or not there is effluent (so that pressing the switch results in $D_{1}$ being 15 , and not pressing it result in $D_{1}$ being 0 ) then it seems reasonable to take 0 and 15 as the only values. On the other hand, if the defendant can control the amount of effluent, then taking the range of values to include every number between 0 and 15 seems more reasonable. 
Perhaps not surprisingly, this issue is relevant to the NESS test as well, for the same reason. If the only possible values of $D_{1}$ are 0 or 15 , then there is no set $\mathbf{S}$ including $D_{2}=13$ that is sufficient for the injury such that $D_{2}=13$ is necessary. On the other hand, if $D_{1}=4$ is a possible event, then there is such a set.

The second problem raised above, the question of which events can go into $\mathrm{S}$, is easy to deal with, by simply making the set explicit. Of course, as the examples above suggest, the choice of events will have an impact on what counts as a cause, but that is arguably appropriate. Recall that causal models deal with this issue by making explicit the signature, that is, the set of variables and their possible values. This gives us a set of primitive events of the form $X=x$. More complicated events can be formed as Boolean combinations of primitive events, but it may also be reasonable to restrict $\mathbf{S}$ to consist of only primitive events.

The first problem, defining sufficient cause, seems more serious. I believe that a formal definition will require some of the machinery of causal models, including structural equations. (This point echoes criticisms of NESS and related approaches by Pearl [2000, pp. 314-315].) In [Halpern 2008], an approach to defining causality in the spirit of Wright's definition is sketched, using the machinery of causal models. The approach delivers reasonable answers in many cases of interest and, indeed, often agrees with the HP definition; however, I have not investigated carefully how it does on problematic examples. ${ }^{12}$

\section{Conclusions}

Perhaps the key point of the HP definition is that causality is relative to a model. This allows us to tailor the model appropriately. Suppose that a drunk 18-year-old gets killed in a single-vehicle road accident. Many people may focus on the drunkenness as the cause of the accident. We all know that you shouldn't drink and drive. But a road engineer may want to focus on the too-sharp curve in the road, a politician may want to focus on the fact that the law allows 18-year-olds to drink, and a psychologist may want to focus on the youth's recent breakup with his girlfriend. ${ }^{13}$ To the extent that causality ascriptions are meant to be guides for future behavior-we ascribe causes so that we know what to do and not to do next time around-it is perfectly reasonable for different communities to focus on different aspects of a situation. Don't drink and drive; don't build roads with sharp curves; don't allow 18-year-olds to drink; and don't drive after you've broken up with your girlfriend may all be useful lessons for different communities to absorb. I view it as an advantage of the HP definition that it can accommodate all these viewpoints easily, by an appropriate choice of endogenous and exogenous variables. (Recall that it is only endogenous variables - the ones that can be manipulated-that can be causes.) The fact that the HP can easily accommodate notions like responsibility and

\footnotetext{
${ }^{12}$ Interestingly, Baldwin and Neufeld [2003] claimed that the NESS test could be formalized using causal models, but did not actually show how, beyond describing some examples. In a later paper [Baldwin and Neufeld 2004], they seem to retract the claim that the NESS test can be formalized using causal models.

${ }^{13}$ This is a variant of an example originally due to Hanson [1958].
} 
blame is further evidence of its usefulness. While it remains to flesh out the case that these notions can be fruitfully applied to legal settings, I am optimistic that this is indeed the case.

Acknowledgments: I thank Steve Sloman for pointing out [Kahneman and Miller 1986], and Joe Gast, Denis Hilton, Chris Hitchcock for interesting discussions on causality.

\section{References}

Alicke, M. (1992). Culpable causation. Journal of Personality and Social Psychology 63, 368-378.

Baldwin, R. A. and E. Neufeld (2003). On the structure model interpretation of Wright's NESS test. In Proc. AI 2003, Lecture Notes in AI, Volume 2671, pp. 9-23.

Baldwin, R. A. and E. Neufeld (2004). The structural model interpretation of the NESS test. In Advances in Artificial Intelligence, Lecture Notes in Computer Science, Volume 3060, pp. 297-307.

Chockler, H., O. Grumberg, and A. Yadgar (2008). Efficient automatic STE refinement using responsibility. In Proc. 14th Conference on Tools and Algorithms for the Construction and Analysis of Systems, pp. 233-248.

Chockler, H. and J. Y. Halpern (2004). Responsibility and blame: A structural-model approach. Journal of A.I. Research 20, 93-115.

Chockler, H., J. Y. Halpern, and O. Kupferman (2008). What causes a system to satisfy a specification? ACM Transactions on Computational Logic 9(3).

Collins, J., N. Hall, and L. A. Paul (Eds.) (2004). Causation and Counterfactuals. Cambridge, Mass.: MIT Press.

Cushman, F. (2009). The role of moral judgment in causal and intentional attribution: What we say or how we think?". Unpublished manuscript.

Cushman, F., J. Knobe, and W. Sinnott-Armstrong (2008). Moral appraisals affect doing/allowing judgments. Cognition 108(1), 281-289.

Friedman, N. and J. Y. Halpern (2001). Plausibility measures and default reasoning. Journal of the ACM 48(4), 648-685.

Galles, D. and J. Pearl (1997). Axioms of causal relevance. Artificial Intelligence 97(1-2), $9-43$.

Gerstenberg, T. and D. Lagnado (2010). Spreading the blame: the allocation of responsibility amongst multiple agents. Cognition 115, 166-171.

Hall, N. (2004). Two concepts of causation. In J. Collins, N. Hall, and L. A. Paul (Eds.), Causation and Counterfactuals. Cambridge, Mass.: MIT Press.

Hall, N. (2007). Structural equations and causation. Philosophical Studies 132, 109-136. 
Halpern, J. Y. (2000). Axiomatizing causal reasoning. Journal of A.I. Research 12, 317-337.

Halpern, J. Y. (2008). Defaults and normality in causal structures. In Principles of Knowledge Representation and Reasoning: Proc. Eleventh International Conference (KR '08), pp. 198-208.

Halpern, J. Y. (2009). From causal models to possible-worlds models of counterfactuals. Unpublished manuscript.

Halpern, J. Y. and C. Hitchcock (2010). Actual causation and the art of modeling. In R. Dechter, H. Geffner, and J. Halpern (Eds.), Causality, Probability, and Heuristics: A Tribute to Judea Pearl, pp. 383-406. London: College Publications.

Halpern, J. Y. and C. Hitchcock (to appear, 2013). Graded causation and defaults. The British Journal for the Philosophy of Science. Available at http://www.cs.cornell.edu/home/halpern/papers/normality.pdf.

Halpern, J. Y. and J. Pearl (2005a). Causes and explanations: A structural-model approach. Part I: Causes. British Journal for Philosophy of Science 56(4), 843-887.

Halpern, J. Y. and J. Pearl (2005b). Causes and explanations: A structural-model approach. Part II: Explanations. British Journal for Philosophy of Science 56(4), 889-911.

Hansson, R. N. (1958). Patterns of Discovery. Cambridge, U.K.: Cambridge University Press.

Hart, H. L. A. and T. Honoré (1985). Causation in the Law (second ed.). Oxford, U.K.: Oxford University Press.

Hiddleston, E. (2005). Causal powers. British Journal for Philosophy of Science 56, 27-59.

Hitchcock, C. (2007). Prevention, preemption, and the principle of sufficient reason. Philosophical Review 116, 495-532.

Hitchcock, C. and J. Knobe (2009). Cause and norm. Journal of Philosophy 106, 587-612.

Hume, D. (1748). An Enquiry Concerning Human Understanding. Reprinted by Open Court Press, LaSalle, IL, 1958.

Kahneman, D. and D. T. Miller (1986). Norm theory: comparing reality to its alternatives. Psychological Review 94(2), 136-153.

Kahneman, D. and A. Tversky (1982). The simulation heuristic. In D. Kahneman, P. Slovic, and A. Tversky (Eds.), Judgment Under Incertainty: Heuristics and Biases, pp. 201210. Cambridge/New York: Cambridge University Press.

Katz, L. (1987). Bad Acts and Guilty Minds. University of Chicago Press.

Knobe, J. and B. Fraser (2008). Causal judgment and moral judgment: two experiments. In W. Sinnott-Armstrong (Ed.), Moral Psychology, Volume 2: The Cognitive Science of Morality, pp. 441-447. Cambridge, MA: MIT Press.

Lewis, D. (2000). Causation as influence. Journal of Philosophy XCVII(4), 182-197. 
Lewis, D. K. (1973). Counterfactuals. Cambridge, Mass.: Harvard University Press.

Mackie, J. L. (1965). Causes and conditions. American Philosophical Quarterly 2/4, 261264. Reprinted in E. Sosa and M. Tooley (Eds.), Causation, Oxford University Press, 1993.

Mandel, D. R., D. J. Hilton, and P. Catellani (Eds.) (1985). The Psychology of Counterfactual Thinking. New York: Routledge.

Pearl, J. (1995). Causal diagrams for empirical research. Biometrika 82(4), 669-710.

Pearl, J. (2000). Causality: Models, Reasoning, and Inference. New York: Cambridge University Press.

Roese, N. (1997). Counterfactual thinking. Psychological Bulletin CXXI, 133-148.

Spohn, W. (1988). Ordinal conditional functions: a dynamic theory of epistemic states. In W. Harper and B. Skyrms (Eds.), Causation in Decision, Belief Change, and Statistics, Volume 2, pp. 105-134. Dordrecht, Netherlands: Reidel.

Stalnaker, R. C. (1968). A theory of conditionals. In N. Rescher (Ed.), Studies in Logical Theory, pp. 98-112. Blackwell.

Watson v. Kentucky and Indiana Bridge and Railroad (1910). 137 Kentucky 619. In 126 SW 146.

Wright, R. W. (1985). Causation in tort law. California Law Review 73, 1735-1828.

Wright, R. W. (1988). Causation, responsibility, risk, probability, naked statistics, and proof: Pruning the bramble bush by clarifying the concepts. Iowa Law Review 73, 1001-1077.

Wright, R. W. (2001). Once more into the bramble bush: Duty, causal contribution, and the extent of legal responsibility. Vanderbilt Law Review 54(3), 1071-1132.

Wright, R. W. (2007). Acts and omissions and positive and negative causes. In Emerging Issues in Tort Law, pp. 287-397. Hart Publishing.

Zultan, R., T. Gerstenberg, and D. Lagnado (2012). Finding fault: causality and counterfactuals in group attributions. Cognition 125, 429-440. 